\title{
The Hamiltonians Generating One-Dimensional Discrete-Time Quantum Walks
}

\author{
Tatsuya TATE* \\ Mathematical Institute, Graduate School of Sciences, Tohoku University, Sendai 980-8578, Japan
}

Received June 15, 2013; final version accepted September 18, 2013

\begin{abstract}
An explicit formula of the Hamiltonians generating one-dimensional discrete-time quantum walks is given. The formula is deduced by using the algebraic structure introduced before. The square of the Hamiltonian turns out to be an operator without, essentially, the "coin register," and hence it can be compared with the one-dimensional continuous-time quantum walk. It is shown that, under a limit with respect to a parameter, which expresses the magnitude of the diagonal components of the unitary matrix defining the discrete-time quantum walks, the onedimensional continuous-time quantum walk is obtained from operators defined through the Hamiltonians of the one-dimensional discrete-time quantum walks. Thus, this result can be regarded, in one-dimension, as a partial answer to a problem proposed by Ambainis.
\end{abstract}

KEYWORDS: discrete-time quantum walk, Hamiltonian, continuous-time quantum walk

\section{Introduction}

The notion of discrete-time quantum walks (quantum walks for short) are originally proposed in quantum physics by Aharonov-Davidovich-Zagury [2] and re-discovered in computer science by, for instance, Nayak-Vishwanath [14], Aharonov-Ambainis-Kempe-Vazirani [1], Ambainis-Bach-Nayak-Vishwanath-Watrous [4]. For more historical background, see, for instance [7,10]. The one-dimensional quantum walks, which we are going to consider here, are defined as a non-commutative analogue of the usual random walks on the set of integers, $\mathbb{Z}$, and they are defined as unitary operators on the Hilbert space $\ell^{2}\left(\mathbb{Z}, \mathbb{C}^{2}\right)$ consisting of all square summable $\mathbb{C}^{2}$-valued functions on $\mathbb{Z}$. According to the asymptotic formulas obtained in [17], there is a resemblance between the asymptotic behavior, in a long-time limit, of transition probabilities of one-dimensional quantum walks and the asymptotic behavior, in a highenergy limit, of modulus squares of the Hermite functions on the real line. Thus, it would be reasonable to think onedimensional quantum walks as a discretized, in both of space and time parameters, model for one-dimensional quantum systems. However, concrete formulas of their Hamiltonian had not been made clear. The aim of this paper is to give an explicit formula for the Hamiltonians generating the one-dimensional quantum walks and investigate their properties. As a result, a direct relation between operators defined through the Hamiltonians of the discrete-time quantum walks and the continuous-time quantum walk in one-dimension is obtained.

To describe our main results let us prepare notation. The inner product $\langle\cdot, \cdot\rangle_{\ell^{2}}$ on $\ell^{2}\left(\mathbb{Z}, \mathbb{C}^{2}\right)$ is defined by

$$
\langle f, g\rangle_{\ell^{2}}=\sum_{x \in \mathbb{Z}}\langle f(x), g(x)\rangle_{\mathbb{C}^{2}}, \quad f, g \in \ell^{2}\left(\mathbb{Z}, \mathbb{C}^{2}\right),
$$

where $\langle\cdot, \cdot\rangle_{\mathbb{C}^{2}}$ denotes the standard Hermitian inner product on $\mathbb{C}^{2}$. Let $T$ be a two-by-two special unitary matrix. ${ }^{1}$ The quantum walk associated with $T$, denoted $U(T)$, is defined, as a unitary operator on $\ell^{2}\left(\mathbb{Z}, \mathbb{C}^{2}\right)$, by the formula

$$
U(T)=T P_{1} \tau+T P_{2} \tau^{-1},
$$

where $\tau$ is the shift operator on $\ell^{2}\left(\mathbb{Z}, \mathbb{C}^{2}\right)$ defined by

$$
(\tau f)(x)=f(x-1) \quad\left(f \in \ell^{2}\left(\mathbb{Z}, \mathbb{C}^{2}\right)\right),
$$

and $P_{i}: \mathbb{C}^{2} \rightarrow \mathbb{C}^{2}$ denotes the orthogonal projection onto the one-dimensional subspace $\mathbb{C e}_{i}$ in $\mathbb{C}^{2}$, where $\left\{\mathbf{e}_{1}, \mathbf{e}_{2}\right\}$ denotes the standard basis on $\mathbb{C}^{2}$. Suppose that the special unitary matrix $T$ is given by

2010 Mathematics Subject Classifications: Primary 60F05, Secondary 81Q99.

The author is partially supported by JSPS Grants-in-Aid for Scientific Research (No. 21740117 and No. 25400068).

${ }^{*}$ Corresponding author. E-mail: tate@math.tohoku.ac.jp

${ }^{1}$ For general unitary matrix $T \in U(2)$, we can write $T=\Delta^{1 / 2} T_{1}$ with $T_{1} \in S U(2)$ and $\Delta=\operatorname{det}(T) \in S^{1}$. The corresponding discrete-time quantum walks satisfy $U(T)=\Delta^{1 / 2} U\left(T_{1}\right)$. Thus, we may, without loss of generality, assume $T \in S U(2)$ in this paper. 


$$
T=\left(\begin{array}{cc}
a & b \\
-\bar{b} & \bar{a}
\end{array}\right) \quad \text { with } \quad a b \neq 0 .
$$

We set

$$
s=|a|, \quad r=|b|, \quad \alpha=\frac{a}{|a|}, \quad \beta=\frac{b}{|b|} .
$$

We define the function $\varphi_{s}(\theta)$ in $\theta \in \mathbb{R}$ by

$$
\varphi_{s}(\theta)=\arccos (s \cos \theta) .
$$

For any integer $x$, we define the integrals $I(x), J(x)$ by

$$
I(x)=\frac{1}{2 \pi} \int_{0}^{2 \pi} e^{-i x \theta} \sin \theta \frac{\varphi_{s}(\theta)}{\sin \varphi_{s}(\theta)} d \theta, \quad J(x)=\frac{1}{2 \pi} \int_{0}^{2 \pi} e^{-i x \theta} \frac{\varphi_{s}(\theta)}{\sin \varphi_{s}(\theta)} d \theta .
$$

We define the matrix-valued function $\mathscr{D}_{T}$ on $\mathbb{Z}$ by

$$
\mathscr{D}_{T}(x)=\left(\begin{array}{cc}
s \alpha^{x} I(x) & -i r \alpha^{x+1} \beta J(x+1) \\
i r \alpha^{x-1} \beta^{-1} J(x-1) & -s \alpha^{x} I(x)
\end{array}\right) \quad(x \in \mathbb{Z}) .
$$

We then define the bounded self-adjoint operator $D(T)$ on $\ell^{2}\left(\mathbb{Z}, \mathbb{C}^{2}\right)$ by the formula

$$
D(T)=\sum_{y \in \mathbb{Z}} \mathcal{D}_{T}(y) \tau^{y}
$$

Theorem 1.1. We have $U(T)=e^{i D(T)}$.

That is, the bounded self-adjoint operator $D(T)$ is the Hamiltonian generating the quantum walk $U(T)$. Note that Ko and Yoo [12] also obtained a formula for the Hamiltonian, see the Eqs. (3.3), (3.4) in [12], although, they do not write down a concrete formula on $\ell^{2}\left(\mathbb{Z}, \mathbb{C}^{2}\right)$. The proof of Theorem 1.1 is rather easy, but the method to deduce the formula (1.8) might not be so obvious. However, through the Fourier transform, the diagonalization works well and is easy way ([12]). See $\S 3$. In the present paper, we use the algebraic structure behind one-dimensional quantum walks introduced in [18] to compute the Hamiltonian because it itself would be interesting. This algebraic structure is nothing but a unitary representation of the infinite dihedral group ([15]). See $\$ 2$ in this paper for this viewpoint.

Having obtained the Hamiltonian generating the one-dimensional quantum walk, it would be important to understand its various properties. First, we mention some of simple properties of the operator $D(T)$. The following can be proved in an easy way. (See $\$ 3$ for details.)

- The spectrum, $\operatorname{Spec}(D(T))$, is given by

$$
\operatorname{Spec}(D(T))=[-\arccos (-s),-\arccos (s)] \cup[\arccos (s), \arccos (-s)] .
$$

- $D(T)$ does not have the locality, that is, $D(T)\left(\delta_{x} \otimes \varphi\right)$ has an infinite support, where the function $\delta_{x} \otimes u$ with $x \in \mathbb{Z}$ and $u \in \mathbb{C}^{2}$ is defined by

$$
\left(\delta_{x} \otimes u\right)(y)= \begin{cases}u & (\text { when } y=x), \\ 0 & (\text { when } y \neq x) .\end{cases}
$$

- The square $D(T)^{2}$ is, essentially, an operator acting on scalars (without "the coin register"), that is, we have the following.

$$
D(T)^{2}=\sum_{y \in \mathbb{Z}} \alpha^{y} \mathcal{F}\left(\varphi_{s}^{2}\right)(y) I_{2} \tau^{y}
$$

where $I_{2}$ is the two-by-two identity matrix and $\mathcal{F}: L^{2}\left(S^{1}\right) \rightarrow \ell^{2}(\mathbb{Z})$ is the Fourier transform defined by

$$
\mathcal{F}(k)(x)=\frac{1}{2 \pi} \int_{0}^{2 \pi} e^{-i x \theta} k(\theta) d \theta \quad\left(x \in \mathbb{Z}, \quad k \in L^{2}\left(S^{1}\right)\right),
$$

where $L^{2}\left(S^{1}\right)$ denotes the Hilbert space, with the usual inner product, consisting of all square integrable functions (with respect to the uniform measure) on the unit circle $S^{1}$.

According to the last item of the above, it would be reasonable to consider the operator $\widehat{H}\left(\varphi_{s}\right)$ on the space $\ell^{2}(\mathbb{Z})$ of square summable (scalar) functions on $\mathbb{Z}$ defined by

$$
\widehat{H}\left(\varphi_{s}\right):=\sum_{y} \mathscr{F}\left(\varphi_{s}\right)(y) \tau^{y},
$$

where $\tau$ is now the shift operator on $\ell^{2}(\mathbb{Z})$ defined by the same formula as in (1.2). In [3], Ambainis proposed a problem how discrete- and continuous-time quantum walks can be obtained one from another. Since we found the operators 
$\widehat{H}\left(\varphi_{s}\right)$ acting on $\ell^{2}(\mathbb{Z})$ from the Hamiltonians of the one-dimensional discrete-time quantum walks, it would be interesting to consider some relationships between these operators and the continuous-time quantum walk. Recall that the continuous-time quantum walk, introduced originally by Childs-Farhi-Gutmann [5], is the unitary operator $e^{i t \widehat{H}}$ acting on $\ell^{2}(\mathbb{Z})$, where $\widehat{H}$ denotes the standard symmetric random walk on $\mathbb{Z}$ defined by

$$
\widehat{H}=\frac{1}{2}\left(\tau+\tau^{-1}\right)
$$

Note that we have $\operatorname{Spec}(\widehat{H})=[-1,1]$ while $\operatorname{Spec}\left(\widehat{H}\left(\varphi_{s}\right)\right)=[\arccos (s), \arccos (-s)]$. Therefore, it would be reasonable to scale the function $\varphi_{s}$ as

$$
\psi_{s}(\theta):=\frac{1}{\arcsin (s)} \arcsin (s \cos \theta)=\frac{1}{\arcsin (s)}\left(\frac{\pi}{2}-\varphi_{s}(\theta)\right)
$$

so that $\operatorname{Spec}\left(\widehat{H}\left(\psi_{s}\right)\right)=[-1,1]$, where $\widehat{H}\left(\psi_{s}\right)$ is the operator defined by the formula (1.12) with $\psi_{s}$ replacing $\varphi_{s}$. We then compare the operator $\widehat{H}$ with $\widehat{H}\left(\varphi_{s}\right)$ and $\widehat{H}\left(\psi_{s}\right)$. Note that if we set

$$
\psi_{0}(\theta)=\cos \theta \quad(\theta \in \mathbb{R}),
$$

then one has $\widehat{H}\left(\psi_{0}\right)=\widehat{H}$, and by Taylor's formula for $\arcsin (x)$, we see

$$
\left\|\psi_{s}-\psi_{0}\right\|_{C\left(S^{1}\right)}=O\left(s^{2}\right), \quad\left\|\widehat{H}\left(\psi_{s}\right)-\widehat{H}\left(\psi_{0}\right)\right\|_{\text {op }}=O\left(s^{2}\right) \quad(s \rightarrow 0),
$$

where $\|\cdot\|_{C\left(S^{1}\right)}$ denotes the supremum norm on the algebra $C\left(S^{1}\right)$ consisting of all continuous functions on $S^{1}$ and $\|\cdot\|_{\text {op }}$ denotes the operator norm. Thus, in the above sense, the classical random walk is obtained, in the limit $s$ tending to zero, from the operators $\widehat{H}\left(\psi_{s}\right)$ defined through the Hamiltonians of the discrete-time quantum walks. However, it would be interesting to compare these operators in the weak-limits of the probability distributions. To define these probability distributions, we generalize a little bit our setting up. For any $k \in C\left(S^{1}\right)$, we denote $M(k)$ the multiplication operator on $L^{2}\left(S^{1}\right)$ by $k$. We define the operator $\widehat{H}(k)$ on $\ell^{2}(\mathbb{Z})$ by

$$
\widehat{H}(k)=\mathcal{F} M(k) \mathcal{F}^{*},
$$

where $\mathcal{F}^{*}$ is, with respect to the usual inner product on $\ell^{2}(\mathbb{Z})$, the adjoint operator of the unitary operator $\mathcal{F}$ given explicitly by

$$
\left(\mathcal{F}^{*} f\right)(\theta)=\sum_{x \in \mathbb{Z}} f(x) e^{i x \theta} \quad\left(f \in \ell^{2}(\mathbb{Z})\right)
$$

Note that $\widehat{H}(k)$ with $k=\varphi_{s}$ in (1.17) coincides with the operator defined in (1.12). When $k$ is real-valued, the operator $\widehat{H}(k)$ is self-adjoint, and hence we have the 1-parameter group of unitary operators $e^{i t \widehat{H}(k)}$. For any $t \in \mathbb{R}$, we define the function $p_{t}(k ; x)$ in $x \in \mathbb{Z}$ by

$$
p_{t}(k ; x)=\left|\left\langle e^{i t \widehat{H}(k)} \delta_{0}, \delta_{x}\right\rangle\right|^{2} \quad(x \in \mathbb{Z}),
$$

where $\langle$,$\rangle denotes the standard \ell^{2}$-inner product on $\ell^{2}(\mathbb{Z})$ and $\delta_{x} \in \ell^{2}(\mathbb{Z})$ is defined as

$$
\delta_{x}(y)= \begin{cases}1 & (\text { when } y=x) \\ 0 & \text { (otherwise) }\end{cases}
$$

Note that $\left\{p_{t}(k ; x)\right\}_{x \in \mathbb{Z}}$ is a probability distribution for each $t \in \mathbb{R}$ and each continuous real-valued function $k$ on $S^{1}$. Our next theorem is on the weak limit distributions of the probability measures

$$
d \mu_{t}(k):=\sum_{x \in \mathbb{Z}} p_{t}(k ; x) \delta_{x / t}
$$

on $\mathbb{R}$ for $k=\psi_{0}, k=\varphi_{s}$ and $k=\psi_{s}(0<s<1)$, where $\delta_{\xi}$ for $\xi \in \mathbb{R}$ denotes the Dirac measure at $\xi$.

Theorem 1.2. For any interval I in $\mathbb{R}$, let $\chi_{I}(x)$ denote the indicator function of $I$. We set $\rho(s):=\arcsin (s)$. Then we have the following.

(1) $\underset{t \rightarrow \infty}{\mathrm{w}-\lim _{t}} d \mu_{t}\left(\psi_{0}\right)=\frac{1}{\pi \sqrt{1-x^{2}}} \chi_{(-1,1)}(x) d x$.

(2) $\underset{t \rightarrow \infty}{\mathrm{W}-\lim _{t}} d \mu_{t}\left(\varphi_{s}\right)=\frac{r}{\pi\left(1-x^{2}\right) \sqrt{s^{2}-x^{2}}} \chi_{(-s, s)}(x) d x(0<s<1)$.

(3) $\underset{t \rightarrow \infty}{\mathrm{W}-\lim _{t}} d \mu_{t}\left(\psi_{s}\right)=\frac{\rho(s) r}{\pi\left(1-\rho(s)^{2} x^{2}\right) \sqrt{s^{2}-\rho(s)^{2} x^{2}}} \chi_{(-s / \rho(s), s / \rho(s))}(x) d x(0<s<1)$.

Note that (1) in Theorem 1.2 is well-known ([9]), and the weak limits in (2) and (3) are essentially (scaled) Konno's distribution ([8]), which appears as weak limit distributions of the transition probabilities of the one-dimensional discrete-time quantum walks. By Taylor's formula for $\arcsin (s)$ as $s \rightarrow 0$ one finds that the distribution in the right- 
hand side of (1) is obtained from that in (3) under the weak limit $s \rightarrow 0$. More generally, we have the following.

Theorem 1.3. For $(s, u) \in[0,1) \times[0, \infty)$, we define the probability measures $d \mu_{(s, u)}$ on $\mathbb{R}$ by

$$
d \mu_{(s, u)}= \begin{cases}d \mu_{1 / u}\left(\psi_{s}\right) & (0 \leq s<1,0<u), \\ \frac{\rho(s) \sqrt{1-s^{2}}}{\pi\left(1-\rho(s)^{2} x^{2}\right) \sqrt{s^{2}-\rho(s)^{2} x^{2}}} \chi_{(-s / \rho(s), s / \rho(s))}(x) d x & (0<s<1, u=0), \\ \frac{1}{\pi \sqrt{1-x^{2}}} \chi_{(-1,1)}(x) d x & (s=u=0) .\end{cases}
$$

Then, the map $(s, u) \mapsto d \mu_{(s, u)}$ defines a weakly continuous map from $[0,1) \times[0, \infty)$ to the space of all probability measures on $\mathbb{R}$ with the weak topology. In particular, we have

$$
\underset{(s, 1 / t) \rightarrow(0,0)}{\mathrm{W}-\lim _{t}} d \mu_{t}\left(\psi_{s}\right)=\frac{1}{\pi \sqrt{1-x^{2}}} \chi_{(-1,1)}(x) d x .
$$

Thus, the one-dimensional continuous-time quantum walk is obtained, under the limit $s=|a| \rightarrow 0$, from the scaled operator $\widehat{H}\left(\psi_{s}\right)$ defined through the Hamiltonian $D(T)$ of the one-dimensional discrete-time quantum walk $U(T)$.

We close Introduction by mentioning earlier works closely related to Theorems $1.2,1.3$. To our knowledge, the relationship between weak limits of continuous-time and discrete-time quantum walks was first pointed out by Strauch [16]. In [16], the quantum walk defined by the coin matrix

$$
T=\left(\begin{array}{cc}
\cos \theta & -i \sin \theta \\
-i \sin \theta & \cos \theta
\end{array}\right)
$$

is considered. Strauch constructed a Hamiltonian, under the limit $\theta \rightarrow \pi / 2$, and discovered that the solutions to the corresponding Schrödinger equation are decomposed into two terms, each of which solves the Schrödinger equation for the continuous-time quantum walk. Compared with it, the limit procedure is the same as ours. Both consider the limit $s \rightarrow 0$. But in our approach a one-parameter family of Hamiltonians $H\left(\psi_{s}\right)$ is obtained, before taking a limit $s \rightarrow 0$, which converges, as $s \rightarrow 0$, to the classical symmetric random walk, see (1.16). The relations among weak limit distributions obtained in Theorems 1.2, 1.3 is essentially a consequence of (1.16).

Chisaki-Konno-Segawa-Shikano [6] relates weak limits for discrete-time quantum walks with that for continuoustime quantum walks by introducing the notion of position measurements for discrete-time quantum walks and finaltime-dependent discrete-time quantum walks. Konno-Machida-Wakasa [11] found that both of weak limit distributions for discrete-time and continuous-time quantum walks satisfy certain ordinary differential equations of Fuchs type (Heun equation for discrete-time and Gauss hyper-geometric equation for continuous-time). Then, they relates the both by considering confluent form of Heun equation obtained by taking the limit $s \rightarrow 0$ (in our notation) and a time scaling parameter $\tau \rightarrow \infty$ with constraint $s^{2} \tau=1$. It seems that these works are rather important not just on the relationship between continuous-time and discrete-time quantum walks but on other viewpoints, although our approach seems to be somewhat straightforward at least to relates discrete-time with continuous-time quantum walks.

\section{An Algebraic Structure}

In our previous paper [18], we have introduced an algebraic structure behind the one-dimensional discrete-time quantum walks. Because we need to use this to deduce the Hamiltonian $D(T)$ in the next section, let us begin by recalling this structure. Suppose that we are given two unitary operators $V, W$ on a Hilbert space $\mathscr{H}_{0}$ satisfying the following relations.

(QW1) $W^{2}=-I$.

(QW2) $V W=W V^{-1}$.

We remark that, in [18], we have introduced another unitary operator, $\sigma$, satisfying some relations with $V$ and $W$. However we do not introduce the operator $\sigma$ here because we will not use it. Now, if the positive numbers $s, r$ satisfy $s^{2}+r^{2}=1$, then it turns out that the linear combination

$$
U=s V+r W
$$

is a unitary operator on $\mathscr{H}_{0}$. We have the following examples.

Example 2.1. Let $\mathscr{H}_{0}=\mathbb{C}^{2}$. For any $\alpha, \beta \in S^{1}$, we set

$$
V(\alpha)=\left(\begin{array}{ll}
\alpha & 0 \\
0 & \bar{\alpha}
\end{array}\right), \quad W(\beta)=\left(\begin{array}{cc}
0 & \beta \\
-\bar{\beta} & 0
\end{array}\right) .
$$


Then, the unitary matrices $V=V(\alpha), W=W(\beta)$ satisfy the relations (QW1), (QW2).

Example 2.2. Let $\mathscr{H}_{0}=\ell^{2}\left(\mathbb{Z}, \mathbb{C}^{2}\right)$. We take $\alpha, \beta \in S^{1}$. Let $V, W$ be the quantum walks,

$$
V=U(V(\alpha)), \quad W=U(W(\beta))
$$

defined in (1.1) associated with the unitary matrices $V(\alpha), W(\beta)$ given in Example 2.1. Then $V$ and $W$ satisfy the relations (QW1), (QW2).

We note that for $T \in \mathrm{SU}(2)$ given by (1.3), the quantum walk $U(T)$ is written in the form (2.1) as

$$
U(T)=s U(V(\alpha))+r U(W(\beta)),
$$

where the parameters $s, r, \alpha, \beta$ are defined in (1.4). Furthermore, if we define a unitary matrix $T(z)$ with $z \in S^{1}$ by

$$
T(z)=s V(\alpha z)+r W(\beta \bar{z}),
$$

then, under the Fourier transform $\mathcal{F}: L^{2}\left(S^{1}, \mathbb{C}^{2}\right) \rightarrow \ell^{2}\left(\mathbb{Z}, \mathbb{C}^{2}\right)$, defined by the same formula as in (1.11) for $k \in L^{2}\left(S^{1}, \mathbb{C}^{2}\right), U(T)$ and $T(z)$ are related each other by the formula

$$
\mathcal{F}^{*} U(T) \mathcal{F}=\mathcal{T},
$$

where $\mathcal{T}$ is the multiplication operator defined by

$$
(\mathcal{T} k)(\theta)=T\left(e^{i \theta}\right) k(\theta) \quad\left(k \in L^{2}\left(S^{1}, \mathbb{C}^{2}\right)\right) .
$$

The following is proved in [18].

Proposition 2.3. Suppose that the unitary operators $V, W$ satisfy the relations (QW1) and (QW2). Let $x=$ $\frac{s}{2}\left(V^{*}+V\right), y=\frac{s}{2 i}\left(V-V^{*}\right), w=r W$. Then, the $n$-th power $U^{n}$ of the unitary operator $U$ defined in $(2.1)$ is represented as

$$
U^{n}=T_{n}(x)+(i y+w) U_{n-1}(x),
$$

where $T_{n}(x)$ and $U_{n-1}(x)$ are, respectively, the Chebyshev polynomials of the first kind of degree $n$ and the second kind of degree $n-1$.

Before explaining how to deduce the formula (1.8) of the operator $D(T)$, let us mention the meaning of the algebraic structure $(\mathrm{QW} 1),(\mathrm{QW} 2)$. The relations $(\mathrm{QW} 1)$, (QW2) define a unitary representation of the infinite dihedral group (see, for example, [13]) as follows. The infinite dihedral group $\Gamma$ is a discrete group defined by

$$
\Gamma=\mathbb{Z} \rtimes \mathbb{Z}_{2} \cong \mathbb{Z}_{2} * \mathbb{Z}_{2},
$$

where $\mathbb{Z}_{2}=\{ \pm 1\}$ and it acts on $\mathbb{Z}$ in an obvious manner. As a set, $\Gamma$ is the product $\mathbb{Z} \times \mathbb{Z}_{2}$, and its group structure is given by

$$
(x, \mu)(y, v)=(x+\mu y, \mu \nu) \quad\left((x, \mu),(y, \nu) \in \mathbb{Z} \times \mathbb{Z}_{2}\right) .
$$

Hence the unit is $e:=(0,1)$ and the inverse element of $(x, \mu) \in \mathbb{Z} \times \mathbb{Z}_{2}$ is given by $(-\mu x, \mu)$. We set $a=(1,1)$ and $b=(0,-1)$. Then, $a$ and $b$ generate $\Gamma$ with the relation

$$
a b=b a^{-1}, \quad b^{2}=e .
$$

Let $V$ and $W$ be unitary operators on a Hilbert space $\mathscr{H}_{0}$ satisfying the relations (QW1) and (QW2). Then, we can define a unitary representation $\rho: \Gamma \rightarrow U\left(\mathcal{H}_{0}\right)$ of $\Gamma$, where $U\left(\mathscr{H}_{0}\right)$ denotes the group consisting of all unitary operators on $\mathscr{H}_{0}$, by setting

$$
\rho(a)=V, \quad \rho(b)=-i W .
$$

In particular, by Example 2.2, the quantum walks $V=U(V(\alpha)), W=U(W(\beta))$ define a unitary representation $\left(\ell^{2}\left(\mathbb{Z}, \mathbb{C}^{2}\right), \rho_{\mathrm{QW}}\right)$ of $\Gamma$.

Theorem 2.4. The unitary representation $\left(\ell^{2}\left(\mathbb{Z}, \mathbb{C}^{2}\right), \rho_{\mathrm{QW}}\right)$ of $\Gamma$ so defined is unitarily equivalent to the regular representation $\left(\ell^{2}(\Gamma), R\right)$.

Proof. We first recall that the (right) regular representation $\left(\ell^{2}(\Gamma), R\right)$, where $\ell^{2}(\Gamma)$ is the Hilbert space consisting of all square summable functions on $\Gamma$ with the standard inner product, is defined by the formula

$$
R: \Gamma \rightarrow U\left(\ell^{2}(\Gamma)\right), \quad(R(g) f)(h)=f(h g), \quad f \in \ell^{2}(\Gamma), g, h \in \Gamma .
$$

Let $\delta_{(x, \mu)}$ be an element in $\ell^{2}(\Gamma)$ defined by

$$
\delta_{(x, \mu)}(y, v)= \begin{cases}1 & (\text { when }(y, v)=(x, \mu)), \\ 0 & \text { (otherwise) }\end{cases}
$$

Note that $\left\{\delta_{(x, \mu)} ;(x, \mu) \in \Gamma\right\}$ is an orthonormal basis of $\ell^{2}(\Gamma)$. Then a direct computation shows that the unitary 
operator $u: \ell^{2}(\Gamma) \rightarrow \ell^{2}\left(\mathbb{Z}, \mathbb{C}^{2}\right)$ defined by the formula

$$
u \delta_{(x, 1)}=\alpha^{-x} \delta_{-x} \otimes \mathbf{e}_{1}, \quad u \delta_{(x,-1)}=i \alpha^{-x} \beta^{-1}\left(\delta_{1-x} \otimes \mathbf{e}_{2}\right) \quad(x \in \mathbb{Z})
$$

intertwines two representations $\left(\ell^{2}\left(\mathbb{Z}, \mathbb{C}^{2}\right), \rho_{\mathrm{QW}}\right)$ and $\left(\ell^{2}(\Gamma), R\right)$.

\section{The Hamiltonian $D(T)$}

Let us explain how to deduce the formulas (1.6), (1.7) and (1.8) for the Hamiltonian $D(T)$. As mentioned in $\S 1$, the diagonalization works in this case. Indeed, as in the proof of Theorem 1.1 below, it is enough to compute the matrixvalued function $L(\theta)$ such that $e^{i L(\theta)}=T\left(e^{i \theta}\right)$ where the matrix $T\left(e^{i \theta}\right)$ is defined in (2.2). But the matrix $T\left(e^{i \theta}\right)$ has the eigenvalues $e^{ \pm \varphi_{s}(\theta+\mu)}$. Since $T\left(e^{i \theta}\right)$ is unitary, it is diagonalized by a matrix $B(\theta)$ so that

$$
T\left(e^{i \theta}\right)=B(\theta)\left(\begin{array}{cc}
e^{i \varphi_{s}(\theta+\mu)} & 0 \\
0 & e^{-\varphi_{s}(\theta+\mu)}
\end{array}\right) B(\theta)^{-1}=e^{i L(\theta)}, \quad L(\theta)=B(\theta)\left(\begin{array}{cc}
\varphi_{s}(\theta+\mu) & 0 \\
0 & -\varphi_{s}(\theta+\mu)
\end{array}\right) B(\theta)^{-1} .
$$

This formula with unitary $B(\theta)$ is explained in [12]. [Though $B(\theta)$ can be taken to be unitary, it is not necessary for us.] The diagonalization is an easy way to get $L(\theta)$ just as explained above. But we use Proposition 2.3 here which enables us to obtain directly an effective formula for the $n$-th power of $T\left(e^{i \theta}\right)$. We apply Proposition 2.3 for the matrices $V=V(\alpha z), W=W\left(\beta z^{-1}\right)$ with $\alpha$ and $\beta$ given in (1.4) to get the following formula.

$$
T(z)^{n}=\left(\begin{array}{cc}
p_{n}(\alpha z) & q_{n}(\alpha z) \\
-\overline{q_{n}(\alpha z)} & \overline{p_{n}(\alpha z)}
\end{array}\right) \quad\left(z \in S^{1}\right),
$$

where $p_{n}(z)$ and $q_{n}(z)$ are the Laurent polynomials given by

$$
p_{n}(z)=T_{n}\left(\frac{s}{2}\left(z+z^{-1}\right)\right)+\frac{s}{2}\left(z-z^{-1}\right) U_{n-1}\left(\frac{s}{2}\left(z+z^{-1}\right)\right), \quad q_{n}(z)=r \alpha \beta z^{-1} U_{n-1}\left(\frac{s}{2}\left(z+z^{-1}\right)\right) .
$$

The matrix $T(z)$ is a Laurent polynomial in $z$ and hence $T(z)^{n}$ is also a Laurent polynomial. The above formulas (3.1) and (3.2) states that these Laurent polynomials are written in terms of the Chebyshev polynomials. Setting $\alpha=e^{i \mu}$, $z=e^{i \theta}$, and using the function $\varphi_{s}(\theta)$ defined in (1.5), we see that

$$
p_{n}(\alpha z)=\cos \left(n \varphi_{s}(\theta+\mu)\right)+i s \sin (\theta+\mu) \frac{\sin \left(n \varphi_{s}(\theta+\mu)\right)}{\sin \varphi_{s}(\theta+\mu)}, \quad q_{n}(\alpha z)=r \beta e^{-i \theta} \frac{\sin \left(n \varphi_{s}(\theta+\mu)\right)}{\sin \varphi_{s}(\theta+\mu)} .
$$

According to the formula (3.3), the functions $p_{n}(\alpha z), q_{n}(\alpha z)$ are differentiable with respect to $n$ at $n=0$. Then, differentiating each component of $T\left(e^{i \theta}\right)^{n}$ in $n$ at $n=0$ and dividing them by $i$, we obtain a matrix $L(\theta)$ defined by

$$
L(\theta)=\frac{\varphi_{s}(\theta+\mu)}{\sin \varphi_{s}(\theta+\mu)}\left(\begin{array}{cc}
s \sin (\theta+\mu) & -i r \beta e^{-i \theta} \\
i r \bar{\beta} e^{i \theta} & -s \sin (\theta+\mu)
\end{array}\right) .
$$

Let $\mathcal{L}$ be the bounded self-adjoint operator on $L^{2}\left(S^{1}, \mathbb{C}^{2}\right)$ defined by

$$
(\mathscr{L} k)(\theta)=L(\theta) k(\theta) \quad\left(k \in L^{2}\left(S^{1}, \mathbb{C}^{2}\right)\right) .
$$

Then, the operator $D(T)$ defined in (1.8) is related to the operator $\mathcal{L}$ by the formula

$$
D(T)=\mathcal{F} \mathscr{L} \mathcal{F}^{*} .
$$

Proof of Theorem 1.1. From (3.5), we see that $e^{i D(T)}=\mathcal{F} e^{i \mathcal{L}} \mathcal{F}^{*}$. Thus, according to (2.3), it is enough to show that $e^{i \mathcal{L}}=\mathcal{T}$. The eigenvalues of $L(\theta)$ defined in (3.4) is $\pm \varphi_{s}(\theta+\mu)$. Then, the matrix $L(\theta)$ is diagonalized as

$$
B(\theta)^{-1} L(\theta) B(\theta)=\left(\begin{array}{cc}
\varphi_{s}(\theta+\mu) & 0 \\
0 & -\varphi_{s}(\theta+\mu)
\end{array}\right)
$$

with the matrix $B(\theta)$ given by

$$
B(\theta)=\left(\begin{array}{cc}
i r \beta e^{-i \theta} & i r \beta e^{-i \theta} \\
s \sin (\theta+\mu)-\sin \varphi_{s}(\theta+\mu) & s \sin (\theta+\mu)+\sin \varphi_{s}(\theta+\mu)
\end{array}\right) .
$$

Then, one can check directly that

$$
e^{i L(\theta)}=B(\theta)\left(\begin{array}{cc}
e^{i \varphi_{s}(\theta+\mu)} & 0 \\
0 & e^{-i \varphi_{s}(\theta+\mu)}
\end{array}\right) B(\theta)^{-1}=T\left(e^{i \theta}\right),
$$


which shows $e^{i \mathcal{L}}=\mathcal{T}$ and hence Theorem 1.1.

Since the spectrum of the operator $D(T)$ coincides with that of $\mathcal{L}$ and the latter is given by the union of the images of two functions $\theta \mapsto \pm \varphi_{s}(\theta+\mu)$, we have (1.9). (1.10) is a consequence of the fact that $L(\theta)^{2}=\varphi_{s}^{2}(\theta+\mu) I_{2}$.

\section{Limit Theorems}

Let $k$ be a smooth real-valued function on $S^{1}$ and consider, for each $t \in \mathbb{R}$, the measure $d \mu_{t}(k)$ defined in (1.19). To prove Theorem 1.2, we use the characteristic function $E_{t}(k ; \xi)$ of $d \mu_{t}(k)$, which is explicitly given by the formula

$$
E_{t}(k ; \xi)=\sum_{x \in \mathbb{Z}} p_{t}(k ; x) e^{i x \xi / t} \quad(\xi \in \mathbb{R})
$$

Let us rewrite the function $E_{t}(k ; \xi)$ in a useful form. Recall that the convolution of two continuous functions $f, g$ on $S^{1}$ is defined by

$$
(f * g)(\theta)=\frac{1}{2 \pi} \int_{0}^{2 \pi} f(r) g(\theta-r) d r .
$$

If $f$ is a smooth function on $S^{1}$, then the Fourier series

$$
f(\theta)=\sum_{x \in \mathbb{Z}} \mathcal{F}(f)(x) e^{i x \theta}
$$

of $f$ converges to $f$ itself uniformly in $\theta$, and hence, for $f, g \in C^{\infty}\left(S^{1}\right)$,

$$
(f * g)(\theta)=\sum_{y \in \mathbb{Z}} \mathcal{F}(f)(y) \mathcal{F}(g)(y) e^{i y \theta},
$$

which converges uniformly in $\theta$. We set $\iota(f)(\theta):=\overline{f(-\theta)}$ so that $\mathcal{F}(\iota(f))(x)=\overline{\mathcal{F}(f)(x)}$ and

$$
(f * \iota(f))(\xi)=\sum_{x \in \mathbb{Z}}|\mathcal{F}(f)(x)|^{2} e^{i x \xi} .
$$

Now we set

$$
f(\theta)=\sum_{x \in \mathbb{Z}}\left\langle e^{i t \widehat{H}(k)} \delta_{0}, \delta_{x}\right\rangle e^{i x \theta}
$$

which is well-defined in $L^{2}\left(S^{1}\right)$. Since $e^{i t \widehat{H}(k)}=\mathcal{F} e^{i t M(k)} \mathcal{F}^{*}$ and $\mathcal{F}^{*} \delta_{x}=e_{x}$, where $e_{x} \in L^{2}\left(S^{1}\right)$ is defined by $e_{x}(\theta)=e^{i x \theta}$, we have $\left\langle e^{i t \widehat{H}(k)} \delta_{0}, \delta_{x}\right\rangle=\left\langle e^{i t M(k)} e_{0}, e_{x}\right\rangle$ and hence $f(\theta)=e^{i t M(k)} e_{0}=e^{i t k(\theta)}$. Thus $f$ is a smooth function on $S^{1}$, and by (1.18), (4.1), (4.2), we see

$$
E_{t}(k ; t \xi)=(f * \iota(f))(\xi)=\frac{1}{2 \pi} \int_{0}^{2 \pi} e^{i t[k(\theta)-k(\theta-\xi)]} d \theta
$$

Proposition 4.1. Let $k$ be a smooth real-valued function on $S^{1}$. Then, for each $\xi \in \mathbb{R}$, we have

$$
\lim _{t \rightarrow \infty} E_{t}(k ; \xi)=\frac{1}{2 \pi} \int_{0}^{2 \pi} e^{i \xi k^{\prime}(\theta)} d \theta
$$

Proof. By (4.3) and Taylor's formula, we have

$$
E_{t}(k ; \xi)=\frac{1}{2 \pi} \int_{0}^{2 \pi} e^{i t[k(\theta)-k(\theta-\xi / t)]} d \theta=\frac{1}{2 \pi} \int_{0}^{2 \pi} e^{i t\left[\xi k^{\prime}(\theta) / t+O\left(1 / t^{2}\right)\right]} d \theta \rightarrow \frac{1}{2 \pi} \int_{0}^{2 \pi} e^{i \xi k^{\prime}(\theta)} d \theta
$$

as $t \rightarrow \infty$, which completes the proof.

Proof of Theorem 1.2. The items (1), (2), and (3) in Theorem 1.2 are easily proved by using Proposition 4.1 with $k=\psi_{0}$ for (1), $k=\varphi_{s}$ for (2) and $k=\psi_{s}$ for (3), respectively.

Proof of Theorem 1.3. Let $\left(s_{o}, u_{o}\right) \in[0,1) \times[0, \infty)$. First assume that $u_{o}>0$. We $\operatorname{set} \arcsin (s)=s\left(1+s^{2} a(s)\right)$ with a smooth function $a(s)$ bounded on $0 \leq s \leq 1 / 2$. We have

$$
\psi_{s}(\theta)=\cos \theta \frac{1+s^{2} a(s \cos \theta)}{1+s^{2} a(s)},
$$

which shows that the function $\psi_{s}(\theta)$ is continuous for $(s, \theta)$ with $0 \leq s$ and $\theta \in \mathbb{R}$. Thus, the characteristic function

$$
E_{t}\left(\psi_{s} ; \xi\right)=\frac{1}{2 \pi} \int_{0}^{2 \pi} e^{i t\left[\psi_{s}(\theta)-\psi_{s}(\theta-\xi / t)\right]} d \theta
$$


is continuous in $s, t, \xi$ even when $s=0$. Hence the weak continuity of $d \mu_{(s, u)}$ at $\left(s_{o}, u_{o}\right)$ is obvious when $u_{o}>0$. Let us prove that $d \mu_{(s, u)}$ is weakly continuous at $\left(s_{o}, 0\right)$. Suppose first that $s_{o}>0$. Obviously we have w-lim $d \mu_{s, 0}=d \mu_{s_{o}, 0}$. Let $s, u>0$ and set $t=1 / u$. Then, we have

$$
\left|E_{t}\left(\psi_{s} ; \xi\right)-\frac{1}{2 \pi} \int_{0}^{2 \pi} e^{i \xi \psi_{s_{o}}^{\prime}(\theta)} d \theta\right| \leq \frac{1}{2 \pi} \int_{0}^{2 \pi}\left|e^{i \xi\left(\psi_{s}^{\prime}(\theta)-\psi_{s_{o}}^{\prime}(\theta)\right)-i\left(\xi^{2} / t\right) \int_{0}^{1}(1-r) \psi_{s}^{\prime \prime}(\theta-r \xi / t) d r}-1\right| d \theta .
$$

By (4.4), we have

$$
\psi_{s}^{\prime}(\theta)=-\sin \theta\left(1+O\left(s^{2}\right)\right), \quad \psi_{s}^{\prime \prime}(\theta)=-\cos \theta\left(1+O\left(s^{2}\right)\right) .
$$

Hence the right-hand side of (4.5) tends to zero as $(s, 1 / t) \rightarrow\left(s_{o}, 0\right)$. Since the characteristic function of the measure $d \mu_{\left(s_{o}, 0\right)}$ is given by

$$
\xi \mapsto \frac{1}{2 \pi} \int_{0}^{2 \pi} e^{i \xi \psi_{s_{o}}^{\prime}(\theta)} d \theta,
$$

we see that $d \mu_{(s, u)}$ is weakly continuous at $\left(s_{o}, 0\right)$. Next, let us prove the continuity at $(0,0)$. Obviously we have $\mathrm{w}-\lim _{s \rightarrow 0} d \mu_{(s, 0)}=d \mu_{(0,0)}$. Now let $s, u>0$ and we set $t=1 / u$. Then, again by Taylor's formula, we obtain

$$
\left|e^{i t\left[\psi_{s}(\theta)-\psi_{s}(\theta-\xi / t)\right]}-e^{-i \xi \sin \theta}\right| \leq C\left(s^{2}+1 / t\right),
$$

where the positive constant $C$ can be chosen uniformly in $\theta \in \mathbb{R}$ and locally uniformly in $\xi \in \mathbb{R}$. From this, we have

$$
E_{t}\left(\psi_{s} ; \xi\right)=\frac{1}{2 \pi} \int_{0}^{2 \pi} e^{-i \xi \sin \theta} d \theta+O\left(s^{2}+1 / t\right)=\frac{1}{\pi} \int_{-1}^{1} \frac{\cos (\xi x)}{\sqrt{1-x^{2}}} d x+O\left(s^{2}+1 / t\right)
$$

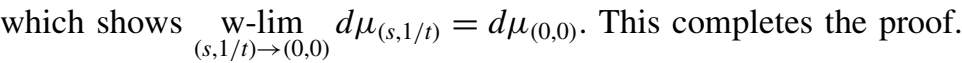

\section{Acknowledgments}

The fact that the algebraic structure introduced in [18] defines a unitary representation of the infinite dihedral group, which is discussed in $\$ 2$ in detail, is suggested to the author by Professor Nobuaki Obata. The author would like to express his special thanks to Professor Obata. The author also would like to thank the referees for the careful reading of the first version of the present paper and for informing us about the works $[6,11,12,16]$.

\section{REFERENCES}

[1] Aharonov, D., Ambainis, A., Kempe, J., and Vazirani, U., “Quantum walks on graphs," Proc. 33rd Annual ACM Symposium on Theory of Computing, 55-59 (2001).

[2] Aharonov, Y., Davidovich, L., and Zagury, N., "Quantum random walks," Phys. Rev. A, 48(2): 1687-1690 (1993).

[3] Ambainis, A., "Quantum walks and their algorithmic applications," Int. J. Quantum Inf., 1(4): 507-518 (2003).

[4] Ambainis, A., Bach, E., Nayak, A., Vishwanath, A., and Watrous, J., "One-dimensional quantum walks," Proc. 33rd Annual ACM Symposium on Theory of Computing, 37-49 (2001).

[5] Childs, A. M., Farhi, E., and Gutmann, S., "An example of the difference between quantum and classical random walks," Quantum Inf. Process., 1: 35-43 (2002).

[6] Chisaki, K., Konno, N., Segawa, E., and Shikano, Y., "Crossovers induced by discrete-time quantum walks," Quantum Inf. Comput., 11: 741-760 (2011).

[7] Kempe, J., "Quantum random walks - an introductory overview," Contemporary Phys., 44(4): 307-327 (2003).

[8] Konno, N., "A new type of limit theorems for the one-dimensional quantum random walk," J. Math. Soc. Jpn., 57: 1179-1195 (2005).

[9] Konno, N., "Limit theorem for continuous-time quantum walk on the line," Phys. Rev. E, 72: 026113 (2005).

[10] Konno, N., Quantum walks, Quantum potential theory, 309-452, Lecture Note in Math., 1954, Springer, Berline (2008).

[11] Konno, N., Machida, T., and Wakasa, T., "The Heun differential equation and the Gauss differential equation related to quantum walks," Yokohama Math. J., 58: 53-63 (2012).

[12] Ko, C. K., and Yoo, H. J., "The generator and quantum Markov semigroup for quantum walks," Kodai Math. J., 36: 363-385 (2013).

[13] Magnus, W., Karrass, A., and Solitar, D., Combinatorial Group Theory, Presentations of Groups in Terms of Generators and Relations, Pure and Applied Math. 13, John Wiley \& Sons, Inc. (1966).

[14] Nayak, A., and Vishwanath, A., Quantum walk on the line, preprint (arXiv: quant-ph/0010117).

[15] Obata, N., Private communication.

[16] Strauch, F. W., "Connecting the discrete- and continuous-time quantum walks," Phys. Rev. A, 74: 030301 (2006).

[17] Sunada, T., and Tata, T., "Asymptotic behavior of quantum walks on the line," J. Funct. Anal., 262: $2608-2645$ (2012).

[18] Tate, T., An algebraic structure for one-dimensional quantum walks and a new proof of the weak limit theorem, preprint (arXiv: 1210.0631), To appear in Infinite Dimensional Analysis, Quantum Probability and Related Topics. 